\title{
HIGH-l RYDBERG LINES OF Fe I IN THE ATMOS SPECTRA:
}

\author{
$4 f-5 g, 5 g-6 h \ldots$ \\ WILLIAM G. SCHOENFELD and EDWARD S. CHANG \\ Department of Physics and Astronomy, University of Massachusetts, \\ Amherst, MA 01003, U.S.A. \\ and \\ MURRAY GELLER \\ Atmospheric and Cometary Sciences Section, Jet Propulsion Laboratory, \\ Pasadena, CA 91109, U.S.A.
}

\begin{abstract}
We have identified the Fe I $4 f-5 g$ lines at $4 \mu \mathrm{m}$ in the $A T M O S$ solar spectra. Using the polarization model as previously applied to silicon, we predict and identify the $5 g-6 h$ lines at $7 \mu \mathrm{m}$. Additional absorption features at 2.5 and $12 \mu \mathrm{m}$ are also shown to be due to high-l Rydberg transitions in $\mathrm{Fe} \mathrm{I}$.
\end{abstract}

Key words: atomic processes - Fe I - infrared: stars - line: identification

\section{Solar Rydberg Lines}

The polarization theory for a non-penetrating Rydberg atom (Edlén, 1964) implies that every atom has highly excited levels whose energies are nearly hydrogenic. As is well known, transitions between Rydberg levels of neutral atoms are found in the infrared; and fortunately the ATMOS spectrum spans most of the region of interest. Thus, any atom with sufficiently high stellar abundance and a relatively low ionization potential should produce Rydberg lines strong enough to be observed in the uncontaminated ATMOS spectra. Previously identified hydrogen-like transitions in the solar spectrum have been attributed to Al I, Si I (Chang, 1984), as well as the extensively-studied $12 \mu \mathrm{m}$ lines of magnesium (Chang and Noyes, 1983). By virtue of its solar abundance and the relative complexity of its core configuration, iron is expected to produce infrared lines that are approximately as strong and far more numerous than those of the simple core atoms, whose Rydberg lines have already been identified.

\section{Rydberg Levels in Fe I}

The Rydberg electron in Fe I is loosely coupled to the core $\left(1 s^{2} \ldots 3 d^{6} 4 s^{1}\right)$, whose lowest term is ${ }^{6} D$. This term gives rise to five distinct core fine structure levels whose angular momentum $\left(j_{c}\right)$ couples to the Rydberg electron's orbital angular momentum in the $j l$ coupling scheme. For non-penetrating Rydberg states $(l \geq 4)$, the quantum numbers $j_{c}, n$ and $l$, along with $K\left(\vec{K}=\overrightarrow{j_{c}}+\vec{l}\right)$, are sufficient to completely specify the Rydberg levels. For partially penetrating $f$ electrons $(l=3)$, the fine structure due to the spin of the Rydberg electron is large enough to necessitate the use of the $J K$ coupling scheme (Cowan and Andrew, 1965). The addition of the spin of the Rydberg electron to form the total angular momentum $J(\vec{J}=\vec{K}+\vec{s})$, produces pairs of closely spaced levels. 


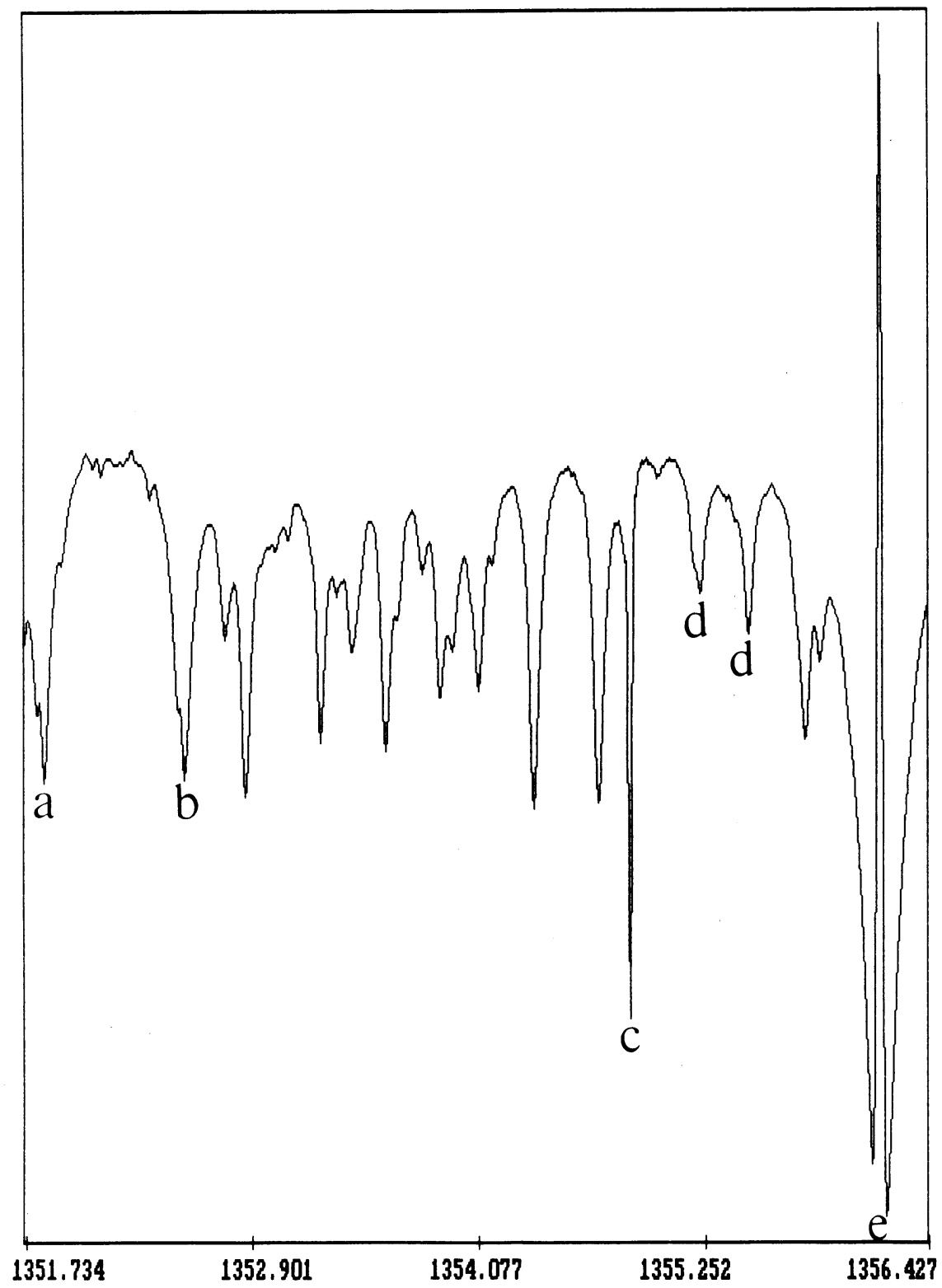

Fig. 1. 5g-6h Rydberg lines in the ATMOS solar spectra. a) aluminum, b) silicon, c) satellite gas, d) carbon, e) magnesium, all other lines are attributed to iron. 
In the simple version of the polarization model for non-spherical cores (Chang, 1984), the energy of the Rydberg level is

$$
E_{n l j_{c} K}=I P-\frac{\mathrm{Ryd}}{\mathrm{n}^{2}}+E_{j_{c}}^{\mathrm{core}}-\alpha\left\langle\frac{1}{r^{4}}\right\rangle_{n l}-2 Q\left\langle j_{c} l K\left\|C^{2}\right\| j_{c} l K\right\rangle\left\langle\frac{1}{r^{3}}\right\rangle_{n l} .
$$

In this expression $I P$ is the ionization potential of the atom, while $E_{j_{c}}^{\text {core }}$ represents the fine-structure energy of the core. The isotropic dipole polarizability $\alpha$, is the first multipole polarizability that is induced in the core by the Rydberg electron. This distortion of the electron cloud lowers the overall energy of the level. The last term represents the electrostatic interaction between the permanent quadrupole moment of the core $Q$, and the Rydberg electron. Both the sign and magnitude of this interaction depend explicitly on $j_{c}, l$ and $K$ via the angular portion of the matrix element. All core fine structure levels with $j_{c}>1 / 2$ will have a non-zero quadrupole interaction. As usual,

$$
\left\langle\frac{1}{r^{3}}\right\rangle_{n l} \text { and }\left\langle\frac{1}{r^{4}}\right\rangle_{n l}
$$

are the hydrogenic radial expectation values over the Rydberg electron wavefunction. These expectation values are simple functions of $n$ and $l$. The simplicity of the polarization formula allows $\alpha$ and $Q$ to be regarded as fitting parameters used to model the interaction of the Rydberg electron with the atomic core.

\section{Line Identification}

Since the $4 f$ levels in iron have recently been determined (Johansson and Learner, 1990 ), and the $4 f-5 g$ transitions are known to occur around $4 \mu \mathrm{m}$, we searched for pairs of lines of nearly equal strengths, whose splittings matched the splittings of the $4 f$ pairs. With tentative identifications for the strongest lines, we were able to fit the model to the observed transition wavenumbers in order to determine the values of the polarization parameters. With reliable values for $\alpha$ and $Q$, we were able to predict and identify all the remaining Rydberg lines.

The laboratory confirmation of the $4 f-5 g$ lines of iron are presented by Johansson et al. (1993), in this volume, so we shall only mention that we have identified 83 absorption lines between $2543 \mathrm{~cm}^{-1}$ and $2582 \mathrm{~cm}^{-1}$. Turning to the $5 g-6 h$ array, we have calculated the positions of all the allowed transitions, and located those that can be observed in the ATMOS spectrum, 31 in all. Most of these are visible in Figure 1, along with Rydberg transitions of other elements. Table 1 identifies the $5 g-6 h$ transitions in Fe I by providing the theoretically predicted line positions along with those actually observed. With a few exceptions, the differences between the two are less than $0.07 \mathrm{~cm}^{-1}$. Many of the weaker Rydberg transitions are blended with other, stronger $5 g-6 h$ Fe I lines. Also included in Table 1 are the calculated hydrogenic line strengths, scaled to facilitate comparison with the observed depths (arbitrary scale). Agreement is excellent, and all observed lines in the region now have identifications except for three very weak features.

As $n$ and $l$ increase, the radial expectation value of the Rydberg electron wavefunction increases. Therefore, the polarization energy that is associated with $\alpha$ 


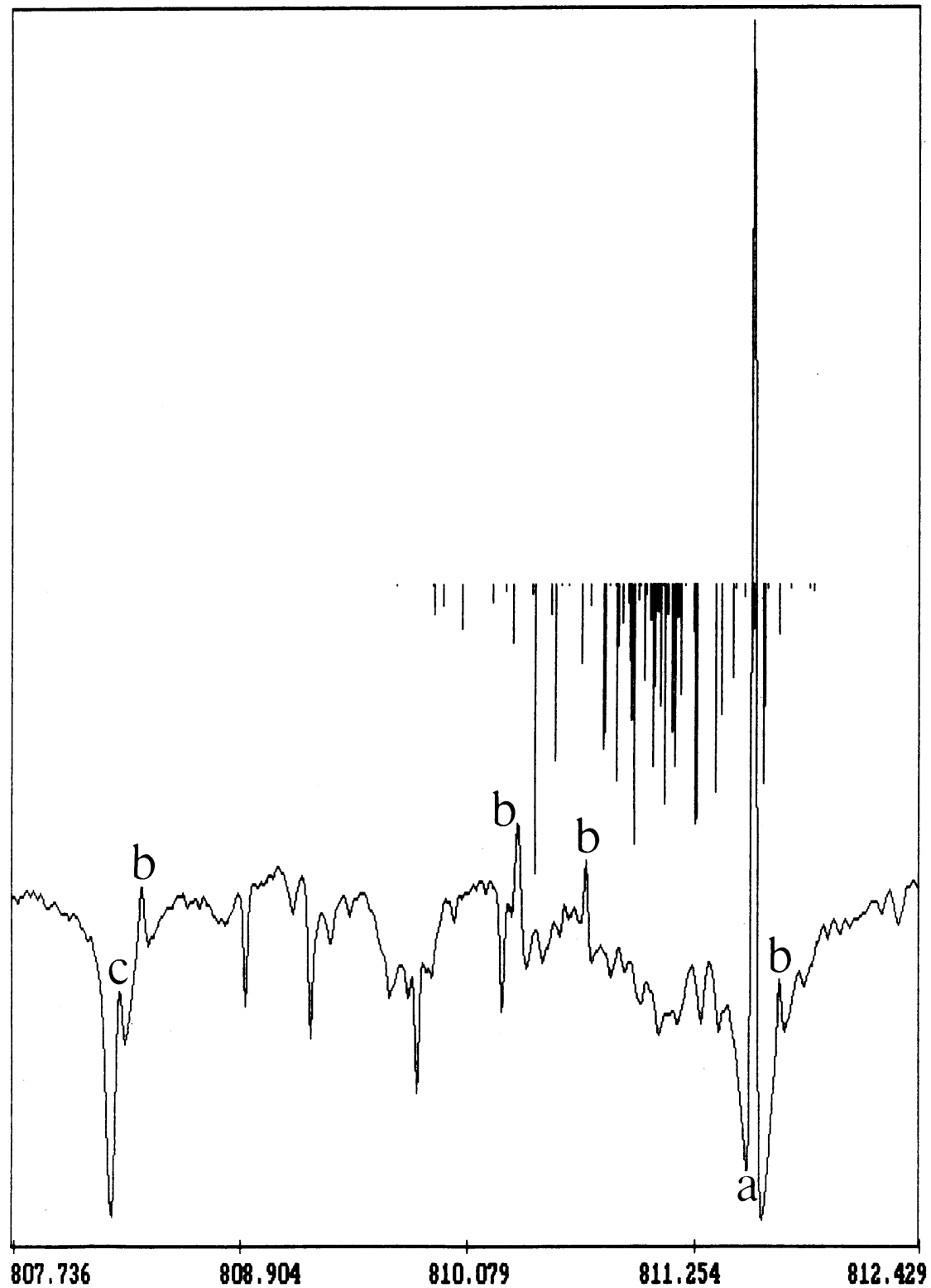

Fig. 2. Rydberg lines at $12 \mu \mathrm{m}$. The positions and the relative strengths of the $6 \boldsymbol{h}-7 \boldsymbol{i}$ lines of Fe I are superimposed above the ATMOS spectra in order to interpret the unknown irregular trough next to the $811 \mathrm{~cm}^{-1}$ of $\mathrm{Mg} \mathrm{I}(\mathrm{a})$. Other lines of interest include b) $6 h-7 i$ emission lines of $\mathrm{Si}$, as well as c) the $n=6 \rightarrow 7$ line of hydrogen. 
TABLE I

5 $g-6 h$ TRANSITIONS IN Fe I

\begin{tabular}{|c|c|c|c|c|c|c|c|}
\hline \multicolumn{3}{|c|}{ IDENTIFICATION } & \multirow{2}{*}{$\begin{array}{c}A T M O S \\
\mathrm{~cm}^{-1}\end{array}$} & \multirow{2}{*}{$\begin{array}{c}\text { THEORY } \\
\text { cm }^{-1}\end{array}$} & \multirow{2}{*}{$\begin{array}{c}\text { LINE } \\
\text { STRENGTH }\end{array}$} & \multirow[t]{2}{*}{$\overline{\text { DEPTH }}$} & \\
\hline$j_{c}$ & $K_{\text {lower }}$ & $K_{\text {upper }}$ & & & & & \\
\hline $9 / 2$ & 0.5 & 0.5 & 1349.731 & 1349.827 & 0.16 & 0.1 & \\
\hline $9 / 2$ & 1.5 & 0.5 & 1350.170 & 1350.229 & 0.23 & 0.3 & \\
\hline $9 / 2$ & 8.5 & 8.5 & 1350.279 & 1350.339 & 0.21 & & bl Si \\
\hline $9 / 2$ & 1.5 & 1.5 & 1350.525 & 1350.689 & 0.26 & & bl Si \\
\hline $9 / 2$ & 2.5 & 3.5 & 1351.400 & 1351.417 & 0.39 & 0.5 & \\
\hline $9 / 2$ & 8.5 & 9.5 & 1351.521 & 1351.648 & 2.24 & 2.2 & \\
\hline $3 / 2$ & 4.5 & 5.5 & 1351.728 & 1351.828 & 0.99 & 1.4 & \\
\hline $9 / 2$ & 2.5 & 2.5 & 1351.937 & 1351.980 & 0.35 & 0.3 & \\
\hline $5 / 2$ & 4.5 & 4.5 & 1352.577 & 1352.606 & 0.17 & & bl Si \\
\hline $5 / 2$ & 4.5 & 5.5 & 1352.778 & 1352.822 & 0.95 & 0.9 & \\
\hline $9 / 2$ & 3.5 & 4.5 & & 1352.895 & 0.58 & & bl \\
\hline $3 / 2$ & 3.5 & 4.5 & & 1352.941 & 0.80 & & bl \\
\hline $5 / 2$ & 5.5 & 6.5 & 1352.885 & 1352.955 & 1.20 & 2.3 & bl \\
\hline $7 / 2$ & 0.5 & 1.5 & & 1352.995 & 0.25 & & bl \\
\hline $5 / 2$ & 3.5 & 3.5 & & 1353.060 & 0.15 & & bl \\
\hline $5 / 2$ & 5.5 & 5.5 & 1353.044 & 1353.083 & 0.15 & 0.1 & bl \\
\hline $7 / 2$ & 7.5 & 7.5 & & 1353.115 & 0.15 & & bl \\
\hline $7 / 2$ & 1.5 & 2.5 & 1353.107 & 1353.149 & 0.35 & 0.2 & \\
\hline $7 / 2$ & 1.5 & 1.5 & & 1353.255 & 0.14 & & bl \\
\hline $7 / 2$ & 7.5 & 8.5 & 1353.276 & 1353.366 & 1.81 & 2.0 & \\
\hline $7 / 2$ & 2.5 & 3.5 & 1353.358 & 1353.404 & 0.50 & & sh \\
\hline $5 / 2$ & 3.5 & 4.5 & 1353.440 & 1353.458 & 0.75 & 1.0 & $\mathrm{bl}$ \\
\hline $9 / 2$ & 3.5 & 3.5 & & 1353.480 & 0.43 & & $\mathrm{bl}$ \\
\hline $7 / 2$ & 2.5 & 2.5 & & 1353.530 & 0.22 & & bl \\
\hline $1 / 2$ & 4.5 & 5.5 & 1353.614 & 1353.710 & 1.02 & 2.0 & $\mathrm{bl}$ \\
\hline $1 / 2$ & 3.5 & 4.5 & & 1353.710 & 0.83 & & bl \\
\hline $7 / 2$ & 3.5 & 4.5 & 1353.679 & 1353.705 & 0.68 & 1.0 & \\
\hline $7 / 2$ & 3.5 & 3.5 & 1353.802 & 1353.828 & 0.28 & 0.5 & $\mathrm{bl}$ \\
\hline $7 / 2$ & 6.5 & 6.5 & & 1353.854 & 0.25 & & bl \\
\hline $5 / 2$ & 2.5 & 2.5 & & 1353.938 & 0.10 & & $\mathrm{bl}$ \\
\hline $7 / 2$ & 6.5 & 7.5 & 1353.90 & 1353.944 & 1.46 & 1.4 & \\
\hline $7 / 2$ & 4.5 & 5.5 & 1353.962 & 1353.970 & 0.89 & 0.9 & \\
\hline $7 / 2$ & 4.5 & 4.5 & & 1354.060 & 0.30 & & bl \\
\hline $7 / 2$ & 5.5 & 6.5 & 1354.096 & 1354.093 & 1.15 & 1.3 & bl \\
\hline $7 / 2$ & 5.5 & 5.5 & & 1354.115 & 0.30 & & bl \\
\hline $9 / 2$ & 7.5 & 7.5 & 1354.167 & 1354.138 & 0.36 & 0.2 & \\
\hline $9 / 2$ & 4.5 & 5.5 & & 1354.379 & 0.82 & & bl \\
\hline $5 / 2$ & 2.5 & 3.5 & & 1354.386 & 0.58 & & bl \\
\hline $5 / 2$ & 6.5 & 7.5 & 1354.381 & 1354.446 & 1.48 & 2.8 & bl \\
\hline $9 / 2$ & 7.5 & 8.5 & 1354.714 & 1354.723 & 1.81 & 2.5 & bl \\
\hline $3 / 2$ & 5.5 & 6.5 & & 1354.740 & 1.23 & & bl \\
\hline $9 / 2$ & 4.5 & 4.5 & 1354.85 & 1354.884 & 0.47 & & sh \\
\hline $5 / 2$ & 6.5 & 6.5 & 1355.017 & 1355.110 & 0.10 & 0.1 & \\
\hline $5 / 2$ & 1.5 & 2.5 & 1355.20 & 1355.241 & 0.45 & & bl C \\
\hline $9 / 2$ & 5.5 & 6.5 & 1355.497 & 1355.499 & 1.10 & & bl C \\
\hline $9 / 2$ & 6.5 & 6.5 & & 1355.739 & 0.45 & & $\mathrm{bl}$ \\
\hline $9 / 2$ & 6.5 & 7.5 & 1355.795 & 1355.796 & 1.42 & 1.6 & bl \\
\hline $9 / 2$ & 5.5 & 5.5 & & 1355.798 & 0.48 & & bl \\
\hline $3 / 2$ & 2.5 & 3.5 & 1355.871 & 1355.854 & 0.64 & 0.8 & \\
\hline
\end{tabular}

bl-blended

sh-shoulder 
decreases. Accordingly, the quadrupole interaction energy that is associated with the non-spherical core atoms decreases in magnitude, thereby decreasing the separation between states of the same $n, l, j_{c}$ but different $K$. The net effect is to push all the lines within a transition array closer together, and also closer to the corresponding line in hydrogen. This is clearly seen in Figure 2, which shows Rydberg lines from a number of different atoms in close proximity to the $n=6 \rightarrow 7$ line in hydrogen. In Fe I, the $4 f-5 g$ transition array is spread out over $40 \mathrm{~cm}^{-1}$, the whole $5 g-6 h$ array is separated by only $6 \mathrm{~cm}^{-1}$, and the $6 h-7 i$ array in Fe I is essentially packed into a $2 \mathrm{~cm}^{-1}$ band. While no specific absorption lines can be associated with the $6 h-7 i$ transition array of $\mathrm{Fe} \mathrm{I}$, it is evident that the closely spaced weak lines combine to form the irregular depression centered at $811 \mathrm{~cm}^{-1}$. The only other possible $\Delta n=1$ transitions expected to be seen in the ATMOS spectra are the $5 f-6 g$ lines. We have examined the region where they should be located, and have found only weak lines $(<1 \%)$. Positive identification remains difficult because the partially penetrating $5 f$ levels are unknown experimentally.

In addition, we have looked for and found some of the weaker $\Delta n=2$ transitions between the $4 f-6 g$ levels at $2.5 \mu \mathrm{m}$. More than fifty identifications have been made, although many of these are blended with stronger CO lines. Other $\Delta n=2$ lines are possible $(n=5 \rightarrow 7,6 \rightarrow 8 \ldots)$, but these are also probably too weak to be seen.

\section{References}

Chang, E.S.: 1984, J.Phys.B 17, L11.

Chang, E.S., and Noyes, R.W.: 1983, Physica Scripta 35, 792.

Cowan, R.D., and Andrew, K.L.: 1965, J. Opt. Soc. Am. 55, 502.

Edlén, B.: 1964, Encyclopedia of Physics vol 27, Springer, Heidelberg.

Johansson, S., and Learner, R.C.M.: 1990, A strophys. J. 354, 775.

Johansson, S., Nave, G., Geller, M., Sauval, A.J., Grevesse, N.: 1993, these proceedings. 